\title{
Dynamic Force Fields zur Präzisionserhöhung von Zeigegeräten
}

\author{
Marcus Specht, Andrea Söter, Jens Gerken, Hans-Christian Jetter, Lorenz \\ Bohrer*, Harald Reiterer \\ Arbeitsgruppe Mensch-Computer Interaktion, Universität Konstanz; *Volkswagen AG
}

\begin{abstract}
Zusammenfassung
Joystick-gestützte Zeigegeräte finden sich in vielen mobilen Informationssystemen, beispielsweise in Smartphones oder auch in Fahrzeugen. Sie sind auf engstem Raum in Systeme integrierbar und hierdurch äußerst flexibel einsetzbar. Sie unterstützen sowohl die Navigation in klassischen Menüstrukturen, wie sie in diesen Szenarien häufig vorzufinden sind, als auch die Steuerung eines Zeigers, beispielsweise für die Interaktion mit Webseiten. Im Vergleich zu Touchpads und -screens sind Joystickgestützte Zeigegeräte auch blind greifbar, mechanisch manipulierbar und bieten spürbare Wiederstände. Durch die Beschränkung auf zumeist 8 digitale Richtungen reduzieren sich jedoch die Präzision und der Bedienkomfort, gerade bei der Steuerung eines Zeigers. In diesem Beitrag wird eine Technik vorgestellt, die durch Analyse der dargestellten Benutzeroberfläche die Präzision und den Bedienkomfort von indirekten Zeigegeräten wie Joysticks signifikant erhöht. Hierzu wird die Nutzerbewegung des Zeigers durch dynamische Kraftfelder so beeinflusst, dass einzelne Oberflächenelemente schneller und fehlerfreier angesteuert werden können, ohne dass die Beeinflussung der Zeigerbewegung als störend empfunden wird. Vor allem bei komplexen Oberflächen mit vielen, gleichzeitig sichtbaren Elementen ist eine möglichst störungs- und irritationsarme Optimierungstechnik von Vorteil. In einem kontrollierten Experiment konnte eine Verbesserung der Usability durch die Dynamic Force Fields statistisch nachgewiesen werden.
\end{abstract}

\section{$1 \quad$ Einleitung}

Das Internet kann als eine der heterogensten Sammlungen graphischer Benutzeroberflächen verstanden werden. Der größte Teil der angebotenen Webseiten ist für die Interaktion mit einer Maus ausgelegt, dem noch dominanten Eingabegerät für Desktopumgebungen. Mit dem technologischen Fortschritt entstanden jedoch Endgeräte, die auch außerhalb von Heim und Büro einen Internetzugang ermöglichen. Je nach Einsatzumgebung werden hierfür Eingabegeräte gewählt, die den zusätzlichen Anforderungen des mobilen Alltags gerecht werden sollen. Eine häufig anzutreffende Klasse sind hierbei Joystick-gestützte Zeigegeräte. Sie sind beispielsweise auf einer Vielzahl von Mobiltelefonen zu finden, unter anderem in Form von 
digitalen vier- bis acht-Wege Schaltern, zur Navigation in Menüs und Steuerung des Zeigers auf Webseiten. Ein weiteres häufiges Einsatzszenario derartiger Eingabegeräte sind die Bedienelemente für die in Fahrzeugen verbauten Informationssysteme (siehe Abbildung 1, links). Im Gegensatz zu einer Maus lassen sie sich auf engstem Raum integrieren und sind zudem vielfältig für die Bedienung des gesamten Informationssystems einsetzbar. Im Gegensatz zu einem Touchpad oder auch einem Touchscreen ist die Bedienhaptik stärker ausgeprägt, was insbesondere auch die blinde Bedienung ermöglicht und gerade für den Einsatz in Fahrzeugen ein wichtiger Aspekt ist. Die Mechanik bzw. Sensorik von Joystick-gestützten Zeigegeräten erlaubt es Benutzern jedoch zumeist nicht, durch unterschiedlich starke Krafteinwirkungen die Geschwindigkeit einer Bewegung zu regulieren. Die Reduzierung auf achtWege schränkt zudem die Präzision und den Bedienkomfort von Zeigersteuerungen ebenfalls deutlich ein. Wird das Eingabegerät während der Fahrt verwendet, können Erschütterungen und Fliehkräfte eine Bedienung weiter erschweren. In einem Kooperationsprojekt mit der Volkswagen AG sollte untersucht werden, inwieweit die Usability solcher Joystickähnlichen Eingabegeräte, insbesondere bei der Bedienung von Webseiten, verbessert werden kann. Dieser Beitrag stellt die Dynamic Force Fields vor, ein Optimierungsverfahren welches durch die Analyse von Webseiteninhalten den Nutzer bei der Ansteuerung dieser durch dynamisch agierende Kraftfelder unterstützt, ohne als störend empfunden zu werden. Nach der Diskussion von verwandten Arbeiten in Kapitel 2 wird die Technik in Kapitel 3 im Detail vorgestellt. In Kapitel 4 werden das Design und die Ergebnisse eines kontrollierten Experiments präsentiert, welches die Usability der Technik statistisch nachweisen konnte.

\section{$2 \quad$ Verwandte Arbeiten}

Um die Interaktionsqualität von Eingabegeräten zu verbessern, können Informationen über das Bewegungsverhalten und die Benutzeroberfläche verwendet werden. Dazu werden Theorien und Modelle aus den Informations- und Ergonomiewissenschaften verwendet, anhand derer Bewegungsabläufe optimiert werden können. Ein in der HCI Gemeinde häufig verwendetes Modell quantifiziert die Schwierigkeit einer Zielbewegung anhand der Distanz zu einem und der Größe von einem Zielobjekt (Fitts 1954). Davon ausgehend können, wenn die Eigenschaften und Besonderheiten der jeweiligen Zeigegeräte und Aufgabenfelder berücksichtigt werden, effiziente und natürlich wirkende Optimierungsverfahren entwickelt werden (MacKenzie 1992). Die in diesem Abschnitt vorgestellten Techniken sollen ein Platzieren des Zeigers mit indirekten Zeigegeräten (Maus, Trackball, Touchpad, Joystick, etc.) auf möglichen Zielen unterstützen. Diese Target Acquisition Performance Techniques manipulieren den visuellen und/oder den motorischen Raum während einer Interaktion. So können beispielsweise Objekte auf der Oberfläche visuell vergrößert werden, sobald sich der Zeiger in ihrer Nähe befindet, um somit einen größeren Zielbereich im visual space anzubieten. Dieses Verhalten kann zwar die Performance steigern (Cockburn \& Brock 2006), ist jedoch ungeeignet für die meisten komplexeren Oberflächen, da sich umgebende Elemente an die Platzveränderungen anpassen müssen. Andere Techniken versuchen Elemente lediglich im motorischen Raum größer wirken zu lassen. Im Falle der Sticky Targets oder Sticky Icons (Worden et al. 1997) wird dies durch dynamische Anpassung des control-to-display (CD) 
gain erreicht. Hierbei wird der Zeiger über möglichen Zielen langsamer (low gain), und diese dadurch von der notwendigen motorischen Bewegung her gesehen größer. Bewegt sich der Zeiger hingegen über nicht aktive Bereiche (whitespace), wird er schneller, ein hoher Skalierungsfaktor (high gain) wird gewählt. Damit kann die Gesamtzeit von Zielbewegungen und die Treffergenauigkeit vor allem bei kleinen Zielen gesteigert werden. Verfahren, die den Zeiger auch orthogonal zur Bewegungsrichtung beeinflussen, verwenden Strukturen ähnlich zu Kraftfeldern, die auf Geschwindigkeit und Richtung des Zeigers einwirken. Diese Force Fields können im Vergleich zu Sticky Targets und klassischen Oberflächen die Performance bei Tapping Tests desweiteren um 7,8\% bis 13,3\% steigern (Ahlström et al. 2006).

Die genannten Verfahren zeigen jedoch Probleme beim Einsatz mit komplexen Oberflächen wie realen Desktopumgebungen. Schneiden Kraftfelder anderer Objekte den Bewegungspfad des Zeigers zum eigentlichen Ziel, so kann ein Abbremsen oder eine Richtungsänderung irritierend und störend wirken. Jede durch Force Fields unterstützte Multiple-ZieleUmgebung (MZU) zählt zu jedem Zeitpunkt $n-1$ mögliche Distraktoren. Ahlström et al. wirkten einem Hängenbleiben in Kraftfeldern entgegen, in dem diese deaktiviert wurden, wenn „six consecutive movements inside the field away from the center“ (Ahlström et al. 2006, 64ff.) registriert wurden. Ein Ziel der in dieser Arbeit vorgestellten Dynamic Force Fields ist es, anstelle dieses heuristischen Ansatzes eine flexiblere Lösung für Multiple-ZielUmgebungen zu finden. Durch Anpassung der Kraftfeldgrößen und -wirkungen unter Berücksichtigung der Oberfläche und Nutzerbewegung soll einerseits die Treffergenauigkeit gesteigert, andererseits eine freie und ungestörte Navigation ermöglicht werden. Dass ein Kompromiss aus homogenen Bewegungsabläufen und Zielunterstützung erstrebenswert ist, zeigte eine Untersuchung zur Benutzerwahrnehmung von $C D$-basierten Verfahren (Mandryk, R. L. \& Gutwin, C. 2008).

\section{3}

\section{Dynamic Force Fields}

Die in dieser Arbeit vorgestellten Dynamic Force Fields (DFF) nutzen Informationen über vorliegende Oberflächen und Benutzerbewegungen, um sich dynamisch deren stetig wechselnden Anforderungen anzupassen. Das Verfahren analysiert zunächst die Benutzeroberfläche und extrahiert Informationen über mögliche Interaktionsziele (Target Aware Technique). So werden im Falle des Webbrowsers geladene Webseiten nach Links, Knöpfen, Texteingabezeilen etc. durchsucht und deren Größe und Position auf der Webseite wird zwischengespeichert. Anhand dieser Daten werden visuell nicht sichtbare Kraftfelder erzeugt und um die Objekte platziert. Die DFFs sind in drei Bereiche gegliedert, die sich in Größe und Funktion unterscheiden können. Für jedes Kraftfeld werden ein äußerer und ein innerer Randbereich sowie ein Zentrum definiert (siehe Abbildung 1 rechts). Für jeden Bereich kann bestimmt werden, wie groß dieser ist, ob der Zeiger darüber schneller (high gain) oder langsamer (low gain) wird und ob die Bewegungsrichtung des Zeigers relativ zum Element angepasst werden soll. Durch diese Fragmentierung, Größen- und Funktionszuordnung können unterschiedliche Szenarien der Optimierung realisiert werden. Um das Ziel dieser Arbeit, möglichst treffsichere und störungsarme Bewegungen auf einer Multiplen-Ziele-Umgebung $(M Z U) \mathrm{zu}$ erreichen, wurden die Bereiche und deren Funktionalität wie folgt definiert. 

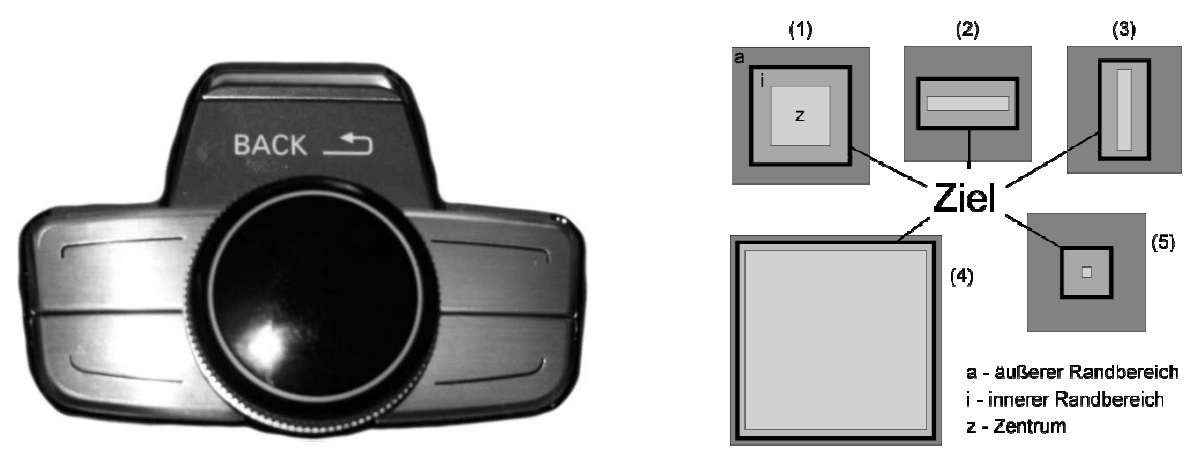

Abbildung 1: (links) 8-fach Schiebe-Dreh-Drücksteller im Fahrzeug, Klicks wurden durch Druck auf den Schieberegler ausgelöst; (rechts) Ziele und deren Kraftfeldbereiche: (1) gleichseitig, (2) breit, (3) hoch, (4) großes Ziel, (5) kleines Ziel.

Die beiden Randbereiche dienen dazu, sowohl die Richtung als auch die Geschwindigkeit des Zeigers zu manipulieren. Die Richtung wird hierbei so verändert, dass der Zeiger in Richtung des Zentrums abgelenkt wird, sobald sich dieser auf dem äußeren Randbereich bewegt (siehe Abbildung 2, links). Der innere Randbereich hat lediglich die Aufgabe, diese Richtungsänderung so lange aufrecht zu erhalten, bis der Zeiger auch visuell von dem Benutzer als auf dem Objekt stehend identifiziert werden kann. Die zu einem gegebenen Zeitpunkt eingeschlagene Richtung bestimmt ebenfalls die Wirkung eines Kraftfeldbereichs. Die richtungsändernde Kraft von Randbereichen wirkt nur wenn sich der Zeiger zum Zentrum des Objekts hinbewegt $\left(<=90^{\circ}\right)$. Extreme Richtungsänderungen und resultierende Irritationen sollen so verhindert werden (siehe Abbildung 2, links). Desweiteren wirkt ein DFF nur auf einen bewegten Zeiger, so dass sich dieser nicht selbstständig ohne Zutun des Nutzers bewegt. Dadurch sollen die Kraftfelder weniger bewusst wahrgenommen und somit eine höhere Akzeptanz erreicht werden. In jedem Fall wird die Richtung des Zeigers nur in den Randbereichen, nicht aber im Zentrum verändert.
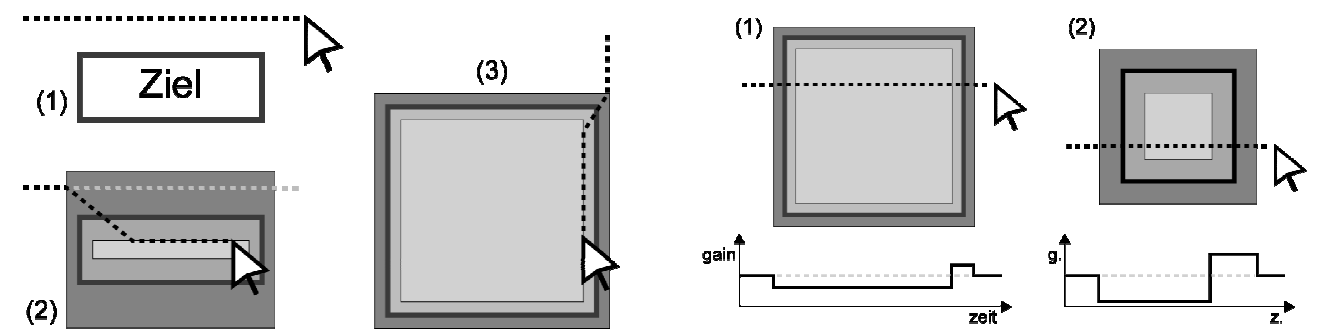

Abbildung 2: (links) Richtungsänderung - (1) ohne Kraftfeld, (2) mit Kraftfeld, (3) geringe Änderung bei großem Ziel; (rechts) CD gain - (1) großes Ziel, geringe Anpassung, (2) kleines Ziel, große Anpassung

Die Größe der Kraftfeldbereiche wird entsprechend der zugewiesenen Oberflächenobjekte relativ gewählt, wobei Höhe und Breite separat behandelt werden. Ist ein Objekt breit, so sind die Randbereiche links und rechts schmal. Je niedriger es ist, desto größer sind die oberen und unteren Bereiche. Das Zentrum nimmt den übrigen inneren Raum ein. So haben sehr 
große Objekte verschwindend geringe Randbereiche, da diese auch ohne Unterstützung gut getroffen werden können (siehe Abbildung 1, rechts). Sehr kleine Objekte haben sehr große, äußere Randbereiche, so dass der Zeiger auch aus größeren Entfernungen angezogen wird.

Der Zeiger wird auf den Randbereichen gebremst (low gain), falls er sich in Richtung des Objekts bewegt. Entfernt er sich vom Zentrum, so wird er auf einem Randbereich kurzzeitig beschleunigt (high gain) um die zuvor „,verlorene“ Zeit wieder aufzuholen. Im Zentrum wird der Zeiger stetig gebremst. Wie stark dieser $C D$ gain den Zeiger bremst oder beschleunigt hängt wieder von der Größe des Objekts ab. Große Objekte bremsen kaum, kleine umso mehr (siehe Abbildung 2, rechts). Der Skalierungswert für die Größe oder den CD gain eines Bereichs wird durch eine Übergangsfunktion bestimmt, welche diesen zwischen einem definierten Minimum und Maximum in Abhängigkeit zur Elementgröße (eb. durch min \& max def.) bestimmt ${ }^{3}$.

Wird nun jedem Objekt ein $D F F$ zugewiesen, ist bei einer realen Anwendung ein Überlappen der Kraftfelder nicht zu vermeiden. Durchfährt der Zeiger solch einen Bereich in dem mehrere Kraftfelder aktiv sind, können unterschiedlich gerichtete Wirkungen störend auf den Bewegungsablauf wirken. Folgende Regeln entscheiden, welches Kraftfeld letztlich wirkt:

- Werden nur äußere Randbereiche geschnitten, kann ein Kraftfeld nur dann wirken, falls alle Wirkungsrichtungen ähnlich sind $\left(<=90^{\circ}\right)$. Es wirkt dann jenes $D F F$, dessen Wirkungsrichtung sich am wenigsten von der Bewegungsrichtung des Nutzers unterscheidet.

- Ein innerer Randbereich sowie das Zentrum wirken, wenn sonst nur äußere Randbereiche geschnitten werden. So kann der Zeiger nicht von zentral überfahrenen Elementen abgelenkt werden.

- Kann durch keine der definierten Regeln eine eindeutige Entscheidung getroffen werden, wirkt keines der geschnittenen Kraftfelder.

Die Aufteilung der Kraftfelder in funktionelle Bereiche, deren relative Größenordnung, das Einbeziehen der Bewegungsrichtung sowie die Regeln zur Auswahl eines wirkenden Kraftfeldes sollen so homogene Bewegungen über die gesamte Oberfläche ermöglichen und dabei das vereinfachte Treffen beliebiger Ziele nachweisbar unterstützen.

\section{$4 \quad$ Evaluation}

Die Usability der dynamischen Kraftfelder sollte im Rahmen eines kontrollierten Experiments überprüft werden. Als Anwendungsszenario wurde hierbei die Bedienung von Webseiten im Fond eines Audi Q7 gewählt. Die Ergebnisse lassen sich aber natürlich auch auf Fahrer (im Stand) und Beifahrer übertragen, da das Bedienelement identisch ist (sh. Abb. 1, rechts). In einem an einen Fitts Tapping Test angelehnten Versuchsaufbau wurden zwei Bedienvarianten der Zeigersteuerung gegenübergestellt. Um eine höhere Praxisrelevanz zu

\footnotetext{
${ }^{3}$ Unter: http://hci.uni-konstanz.de/gerken/DFF_Parameter.pdf finden Sie eine detaillierte Auflistung der Parameter
} 
erreichen, wurde in beide Varianten das Angle Mouse (Wobbrock et al. 2009) Verfahren integriert, das die Zeigergeschwindigkeit dynamisch anhand der Richtungsänderungen des Benutzers variiert und daher keine Informationen über die Benutzeroberfläche benötigt (Target Agnostic Techniques). Wird hierbei lange in dieselbe Richtung navigiert, beschleunigt der Zeiger (high gain). Sobald der Nutzer beginnt, die Richtung zu korrigieren, nimmt das Modell an, dass sich das anvisierte Ziel in der Nähe befindet und die Geschwindigkeit wird verlangsamt (low gain). Variante 1 beinhaltete ausschließlich diese Angle Mouse Optimierung wohingegen Variante 2 diese mit den DFFs kombinierte. Im Folgenden werden zunächst der Testaufbau und die Hypothesen erläutert, bevor die Ergebnisse präsentiert und diskutiert werden.

\subsection{Probanden, Aufgaben, Ablauf \& Design}

Im Rahmen dieser Studie konnte aus organisatorischen Gründen (das Experiment fand in einem realen Fahrzeug statt) nur eine relativ geringe Anzahl an Probanden involviert werden. Um somit die Varianz der Daten aufgrund individueller Unterschiede möglichst gering zu halten, wurde eine möglichst homogene Gruppe von acht Personen im Alter von 16-17 Jahren ausgewählt, sechs davon männlich, zwei weiblich. Die Altersgruppe entspricht der Kernzielgruppe des im Zusammenhang mit dieser Technik entwickelten Webbrowsers - einem Fondpassagier in einem Audi Q7. Zur Messung der Effizienz und Effektivität wurde ein multi-direktionaler Tapping Task eingesetzt. Dieser weicht allerdings von den Standardverfahren, wie in ISO 9241-9, Annex B vorgeschlagen ab, um eine höhere ökologische Validität zu erreichen. Anstelle einzelner abstrakter Elemente, wurde vielmehr eine abstrakte Webseite erstellt, die sich aus Elementen bekannter Webseiten zusammensetzt (vgl. Abbildung 3, links), wie beispielsweise der Hauptnavigation von Spiegel Online oder Steuerungselementen von Youtube.com (Söter 2009). Die Aufgabe der Probanden bestand darin, ein rot aufleuchtendes Element mit Hilfe des Bedienelements so schnell und so genau wie möglich anzusteuern und anzuklicken. Nach dem erfolgreichen Treffen dieses Elements leuchtete das nächste Zielelement rot auf. Diese Aufgabe wurde in fünf Blöcken jeweils 40mal durchgeführt (= fünf mal 40 Trials).
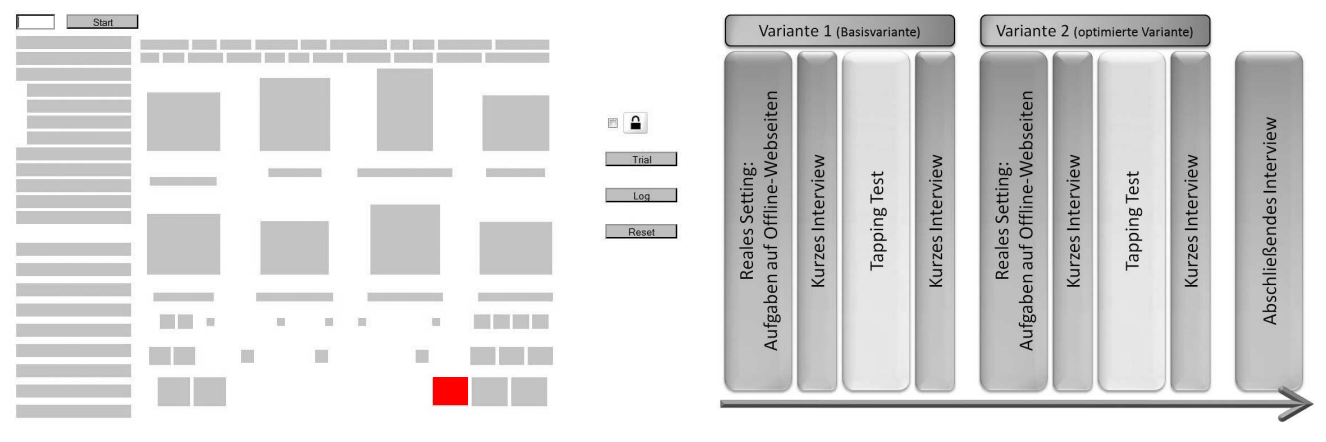

Abbildung 3: Abstrakte Webseite für den Tapping-Test (links),Ablaufdiagramm des Experiments(rechts). 
Die Durchführung erfolgte in einem within-subjects Design, wobei die Reihenfolge der Bedienvarianten ausbalanciert wurde. Die Probanden wurden auf den Wechsel der Varianten nicht hingewiesen, um überprüfen zu können, inwieweit ein Unterschied zwischen diesen bei der Nutzung bemerkt wird (siehe H5). Zunächst bearbeiteten die Probanden Aufgaben auf realen Webseiten. Dies diente zum einen der Eingewöhnung, da die Nutzer zuvor noch kein derartiges Bedienelement benutzt hatten. Zum anderen sollte der im Zusammenhang mit den DFFs entwickelte Webbrowser hier einem qualitativen Usability Test unterzogen werden. Die Ergebnisse dieses Teils der Studie finden sich in Söter (2009). Nach einem kurzen Interview wurde zu dem Tapping Task gewechselt, auf welchen ein weiteres kurzes Interview folgte. Anschließend wurde dieser Ablauf mit der zweiten Variante wiederholt (vgl. Abbildung 3, rechts). Die Kurzinterviews dienten dem Abfragen von positiven und negativen Auffälligkeiten. Mit dem abschließenden Interview sollte geprüft werden, ob die Probanden einen Wechsel zwischen den Varianten bemerkt hatten und wenn ja, inwieweit dieser als positiv oder negativ empfunden wurde.

Jeder Proband nahm zweimal an dem Experiment teil. Zunächst wurde der Test im stehenden Fahrzeugbetrieb durchgeführt. Eine Woche später wurde er während einer Fahrt wiederholt. Dadurch konnte überprüft werden, inwieweit der Kontext der Benutzung Einfluss auf den Nutzen der Technik hat (vgl. Söter 2009).

\subsection{Hypothesen und Variablen}

Die Hypothesen, die im Rahmen der Evaluation überprüft werden sollten, betrafen einerseits die Effektivität und Effizienz der durch die DFFs optimierten Zeigersteuerung. Andererseits sollte untersucht werden, inwieweit die Optimierung den Nutzern auffällt und ob diese als störend empfunden wird. Gemessen wurde die benötigte Zeit pro Trial, die zurückgelegte Distanz in Pixeln (beides zur Effizienzbestimmung), die Anzahl der „Overshoots“, sowie die Anzahl der Fehlklicks (beides zur Effektivitätsbestimmung). Ein Overshoot tritt auf, wenn ein Proband den Zeiger zunächst über das Ziel hinaus bewegte, und somit eine Korrekturbewegung erfolgte. Ein Fehler entspricht einem Klick außerhalb eines vorgegeben Ziels. Entsprechend dem Designziel der DFF, sowohl die Effizienz als auch die Effektivität zu erhöhen, ohne dabei die eigentliche Nutzung negativ $\mathrm{zu}$ beeinträchtigen, lauteten die Forschungshypothesen wie folgt:

H1: Zielelemente können mit Variante 2 (DFF) schneller getroffen werden, als mit Variante 1 (keine DFF).

H2: Die Verwendung von Variante 2 (DFF) führt im Vergleich zu Variante 1 (keine DFF) zu einer geringeren Anzahl an Fehlern.

H3: Die Verwendung von Variante 2 (DFF) führt im Vergleich zu Variante 1 (keine DFF) zu einer geringeren Anzahl an Overshoots.

H4: Die Verwendung von Variante 2 (DFF) führt im Vergleich zu Variante 1 (keine DFF) zu einer Reduzierung der zurückgelegten Strecke.

H5: Die Probanden bemerken keinen Unterschied zwischen den beiden Varianten. 


\subsection{Ergebnisse}

Um den Einfluss durch Lerneffekte zu minimieren, wurden bei der Auswertung der stationären Tests lediglich die letzten beiden der fünf Blöcke des Tapping Tasks gewertet, bei den mobilen Tests jeweils der letzte Block. Mittels Varianzanalyse wurde zunächst der Unterschied zwischen Variante 1 (keine DFF) und der optimierten Variante 2 (DFF) im stationären Einsatz untersucht. Die durchschnittliche Dauer für einen Trial betrug mit Variante 13,10 Sekunden $(\mathrm{s}=0,50)$, wohingegen die Probanden mit Variante 2 mit DFF im Schnitt lediglich 2,89 Sekunden $(\mathrm{s}=0,45)$ benötigten. Dieser Unterschied ist statistisch signifikant $(\mathrm{p}=0,027$. $\mathrm{F}(1,7)=7,746)$ (vgl. Abbildung 4). Auch die Anzahl der Overshoots verminderte sich bei der optimierten Variante 2 im Vergleich zur Basisvariante 1 von 0,439 Overshoots pro Trial ( $\mathrm{s}=$ $0,169)$ auf $0,207(\mathrm{~s}=0,103)$ um knappe $53 \%$ und war somit ebenfalls signifikant geringer (p $=0,002 . \mathrm{F}(1,7)=22,443)(\mathrm{vgl}$. Abbildung 5). Die Fehlerrate war zwar ebenfalls zu Gunsten der DFF geringer (0,019 Fehlern/Trial, $s=0,012$ vs. 0,006 Fehler/Trial, $s=0,009)$, jedoch statistisch nicht signifikant $(\mathrm{p}=0,104 . \mathrm{F}(1,7)=3,5)$. Das gleiche gilt für die zurückgelegte Distanz/Trial (Variante 1: 317,15 Pixel/Trial zurückgelegt $(\mathrm{s}=10,76)$ vs. Variante 2: 316,0 Pixel/Trial $(s=15,02), p=0,769 . F(1,7)=0,094)$. Erwähnenswert ist dennoch, dass sämtliche Maße dieselbe Richtung zu Gunsten der optimierten Variante 2 aufweisen.

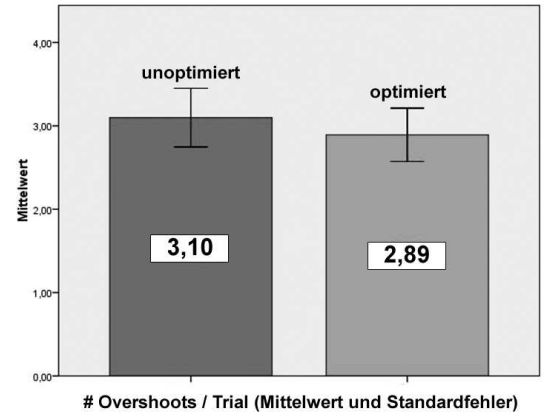

Abbildung 4: Mittelwerte und Streuung der benötigten Zeit pro Trial im stationären Test (Söter 2009).

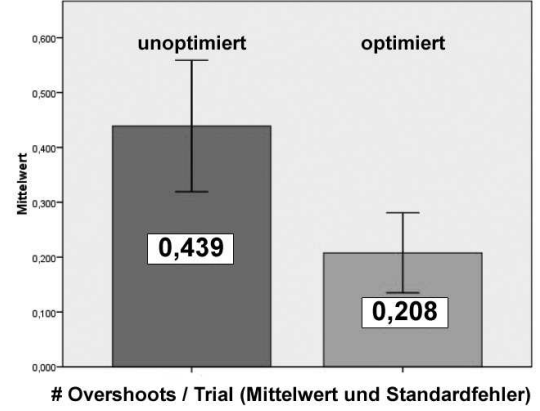

Abbildung 5: Mittelwerte und Streuung der vollführten Overshoots pro Trial im stationären Test (Söter 2009).

Während der Fahrt zeigt sich ein ähnliches Bild. Auch hier ist in Bezug auf sämtliche Maße ein Unterschied zu Gunsten der optimierten Variante festzustellen. Die Zeit, die die Probanden durchschnittlich für einen Trial benötigten verringerte sich von 3,37 Sekunden $(s=0,62)$ bei der Basisvariante auf 3,07 Sekunden $(s=0,44)$ bei der optimierten Variante. Im Gegensatz zum stationären Einsatz ist dieser Unterschied statistisch nicht signifikant $(p=0,103$. $\mathrm{F}(1,7)=3,508$ ). Die Overshoots konnten mit Hilfe der DFFs um 64\% reduziert werden (vgl. Abbildung 7).Während bei der Interaktion mit der Basisvariante noch durchschnittlich 0,522 Overshoots pro Trial $(\mathrm{s}=0,29)$ gemacht wurden, lag dieser Wert mit DFFs bei $0,188(\mathrm{~s}=$ $0,04)$. Dieser Unterschied ist signifikant $(\mathrm{p}=0,012 . \mathrm{F}(1,7)=11,178)$. Die Unterschiede bzgl. der Fehlerrate waren wiederum sehr gering und nicht signifikant $(p=0,184 . F(1,7)=2,172$, Variante 1: 0,05 Fehlern/Trial $(s=0,063)$ vs. Variante 2: 0,013 Fehler/Trial $(s=0,019))$. In 
der mobilen Testumgebung ließ sich jedoch im Gegensatz zu den stationären Tests eine deutliche und statistisch signifikante Verminderung der zurückgelegten Strecke erkennen ( $\mathrm{p}=$ 0,014. $\mathrm{F}(1,7)=10,753)$. Statt 357,08 Pixel $(\mathrm{s}=21,36)$ wie bei der Basisvariante, legten die Probanden mit der optimierten Variante durchschnittlich nur 342,88 Pixel pro Trial (s = 21,52) zurück. Die beobachteten Unterschiede (Geschwindigkeit, Distanz) zwischen stationärem Einsatz und der Fahrt lassen sich nicht vollends abschließend erklären. Ein Grund hierfür kann sicherlich in der geringen Stichprobengröße liegen. Im Falle der zurückgelegten Strecke in Pixeln könnte vermutet werden, dass der Einfluss von störenden Faktoren bei der Fahrt (z.B. Rütteln im Fahrzeug bei holpriger Fahrbahn) den Nutzen der DFFs verstärken konnte.

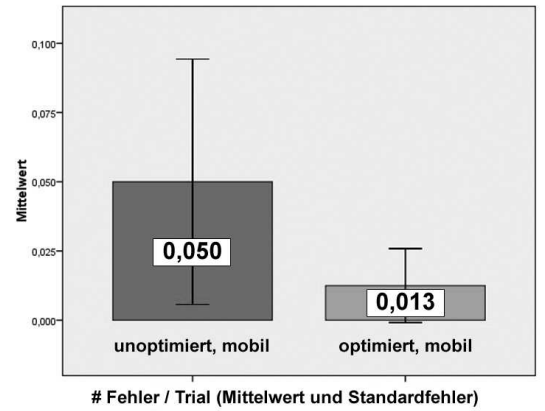

Abbildung 6: Mittelwerte und Streuung der gemachten Fehler pro Trial im mobilen Test (Söter 2009).

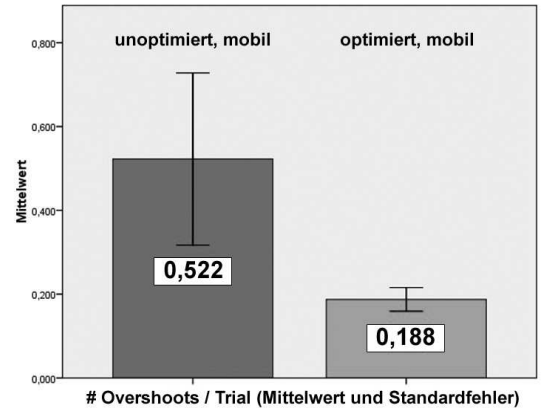

Abbildung 7: Mittelwerte und Streuung der vollführten Overshoots pro Trial im mobilen Test (Söter 2009).

H1 und H4 lässt sich somit nur für den stationären (H1) bzw. mobilen Test (H4) als statistisch signifikant nachweisen. H2 muss hingegen zugunsten der Nullhypothese abgelehnt werden - die Fehlerrate war mit beiden Varianten sehr gering, vermutlich zu „Gunsten“ der Overshoots. Die Anzahl dieser unterscheidet sich sowohl während dem stationären, als auch während dem mobilen Fahrzeugbetrieb statistisch signifikant, womit H3 angenommen werden kann. Unterstützend wirkt, dass der Trend aller Messgrößen in Richtung der DFFs zeigt. Die qualitative Auswertung des Experiments lieferte ebenfalls positive Ergebnisse: Da das Konzept der DFFs dem Benutzer zwar helfen, ihm aber nicht auffallen soll, wurden die Probanden vor der Testreihe nicht auf die zwei zu testenden Varianten hingewiesen. Die Veränderung der Zeigerbewegung bemerkte bei der Interaktion auf realen Webseiten keiner der acht Probanden. Lediglich zwei der Probanden nahmen während des Tapping Tasks eine Veränderung wahr und identifizierten eine künstliche Ablenkung des Zeigers. Diese Probanden gaben jedoch an, die Veränderung als positiv und hilfreich empfunden zu haben. Hypothese H5, welche besagt, dass subjektiv kein Unterschied zwischen den beiden Varianten festgestellt wird, kann somit ebenfalls angenommen werden. Zudem zeigte sich bei der qualitativen Analyse der Nutzung der realen Webseiten (hierzu wurden keine quantitativen Logfiles festgehalten), dass mehrfach beobachtet werden konnte, wie Links mit Hilfe der DFFs in weniger Anläufen und mit weniger Overshoots korrekt getroffen wurden. 


\section{$5 \quad$ Fazit}

Trotz der kleinen Stichprobe haben sich die DFFs bei einem multi-direktionalen Tapping Task statistisch nachweisbar als vorteilhaft erwiesen. Da ebenfalls eine verbesserte Interaktion während qualitativen Nutzerstudien auf realen Webseiten zu beobachten war, kann von einer hohen Praxistauglichkeit der DFFs ausgegangen werden. Dabei kann die Akzeptanz dieser Technik dem Kompromiss aus homogenen Bewegungsabläufen und Zielunterstützung zugeschrieben werden. Ein Nachteil von Target-Aware Verfahren ist jedoch der notwendige Aufwand Informationen wie Position und Größe von Oberflächenkomponenten zu erhalten. Ist dies jedoch gegeben, können die DFFs im Vergleich mit Target-Agnostic Verfahren wie der Angle Mouse nochmals eine deutliche Steigerung der Usability bieten. Zu überprüfen bleibt, inwieweit die DFFs für andere indirekte Zeigegeräte Vorteile bieten können und welche Parameter entsprechende Einsatzszenarien optimal unterstützen.

\section{$6 \quad$ Literaturverzeichnis}

Ahlström, D., Hitz, M. \& Leitner, G. (2006). An Evaluation of Sticky and Force Enhanced Targets in Multi Target Situations. In (Hrsg.): NordiCHI 2006. Oslo: ACM, S. 58-67.

Cockburn, A. \& Brock, P. (2006). Human On-Line Response to Visual and Motor Target Expansion. In Mann, S. \& Gutwin, C. (Hrsg.): Graphics Interface Proceedings 2006. Otawa: A K Peters LTD, S. 81-87.

DIN EN ISO 9241-9, Annex B (2000). Ergonomic requirements for office work with visual display terminals (VDTs) - Part 9: Requirements for non-keyboard input devices. Genf, Schweiz.

Fitts, P.M. (1954). The information capacity of the human motor system in controlling the amplitude of movement. In (Hrsg.): Journal of Experimental Psychology Vol. 47. S. 381-391.

Keyson, D. V. (1997). Dynamic cursor gain and tactual feedback in the capture of cursor movements. In (Hrsg.): Ergonomics. London: Taylor \& Francis, S. 1287-1298

MacKenzie, S. (1992). Fitts' Law as a Research and Design Tool in Human-Computer Interaction. In (Hrsg.): HUMAN-COMPUTER INTERACTION. Taylor \& Francis, S. 91-139

Söter, A. (2009). Theorie und Praxis von Benutzerbefragungen, Benutzungsstudien und Benutzbarkeitstests in der Automobilindustrie. Master-Arbeit, Universität Konstanz.

Wobbrock, J. O., Fogarty, J., Liu, S. S., Kimuro, S. \& Harada, S. (2009). The Angle Mouse: TargetAgnostic Dynamic Gain Adjustment Based on Angular Deviation. In (Hrsg.): Proceedings of the CHI 2009. Boston: ACM, S. 1401-1410.

Worden, A., Walker, N., Bharat, K. \& Hudson, S. (1997). Making computers easier for older adults to use: area cursor and sticky icons. In (Hrsg.): Proceedings of the CHI '97 Conference on Human Factors in Computing System. New York: ACM, S. 266-271.

\section{Kontaktinformationen}

Jens Gerken, Box-D73, Universität Konstanz. E-mail: jens.gerken@uni-konstanz.de 